\title{
Study of Polyurethane Foaming Dynamics Using a Heat Flow Meter
}

\author{
P. Koniorczyk ${ }^{1}$ - M. Trzyna ${ }^{1}$ - J. Zmywaczyk ${ }^{1}$. \\ B. Zygmunt ${ }^{1}$ - M. Preiskorn ${ }^{1}$
}

Received: 16 June 2016 / Accepted: 6 March 2017 / Published online: 15 March 2017 (C) The Author(s) 2017. This article is published with open access at Springerlink.com

\begin{abstract}
This work presents the results of the study concerning the effects of fillers addition on the heat flux density $\dot{q}(t)$ of foaming of polyurethane-polystyrene porous composite (PSUR) and describes the dynamics of this process during the first $600 \mathrm{~s}$. This foaming process resulted in obtaining porous materials that were based on HFC 365/225 blown rigid polyurethane foam (PUR) matrix, which contained thermoplastic expandable polystyrene (EPS) beads as the filler. In PSUR composites, the EPS beads were expanded after being heated to a temperature above the glass transition temperature of EPS and vaporing gas incorporated inside, by using the heat of exothermic reaction of polyol with isocyanate. From the start $(t=0)$ to the end of the PSUR composite foaming process $\left(t=t_{k}\right), \dot{q}(t)$ was measured with the use of the heat flow meter. For the purpose of the study two PUR systems were selected: one with high and one with low heat density of foaming process $q$. EPS beads were selected from the same manufacturer with large and small diameter. The mass fraction of EPS in PSUR foam varied during the measurements. Additionally, a study of volume fractions of expanded EPS phase in PSUR foams as a function of mass fractions of EPS was conducted. In order to verify effects of the EPS addition on the heat flux density during PSUR foaming process, the thermal conductivity measurements were taken.
\end{abstract}

Keywords Composite $\cdot$ Expandable polystyrene $\cdot$ Heat flux density $\cdot$ Rigid polyurethane foam . Thermal conductivity

Selected Papers of the 13th International Symposium on Temperature, Humidity, Moisture and Thermal Measurements in Industry and Science.

\footnotetext{
$凶$ P. Koniorczyk

piotr.koniorczyk@wat.edu.pl

1 Military University of Technology, Warsaw, Poland
} 


\section{Introduction}

Fillers are added to polymers in order to reduce composite cost and to improve processing behavior as well as to modify product properties [1]. Fillers are inexpensive; thus, using them allows making the material cheaper. However, cost reduction is no longer the only reason of fillers addition, nor even the most important. Fillers impact density and other material characteristics such as mechanical and chemical reactivity, thermal conductivity, thermal stability, flame retardancy [2-4]. Fillers are also used in the formulation of foams, although their production is very sensitive to changes in composition and parameters of processing. The inclusion of fillers complicates the process, and careful investigation must be performed to understand the effects that filler incorporation has on material properties. Fillers reduce shrinkage and combustibility of polymer foams. Typical powder fillers used for preparation of rigid polyurethane foams (PUR) are aluminum hydroxide, melamine, starch, talk, chalk, borax [5], calcium carbonate, barium sulfate [6], crystallized silica [7] and nanofillers like bentonite [8] and expanded graphite [9]. It is worth mentioning at this point that the only tool to perform the verification and analysis of the heat transfer mechanism in such foams is the measurement of thermal conductivity $[19,20]$. Since we analyze the finished product, we can only guess what was the course of the exothermic polymerization process as a function of time and how it affected the value of the thermal conductivity of foams.

Cracow University of Technology in cooperation with Military University of Technology (Warsaw, Poland) and HIT Konsulting Sp. z o. o. carried out a research on the method of obtaining new composites based on PUR and expandable polystyrene (EPS). The method of preparing PSUR composite was described in patents $[10,11]$. A thermoplastic granulate containing a blowing agent served as the filler of the PUR matrix. The filler had the softening point below $90^{\circ} \mathrm{C}$. The diameter of beads was in the range between $0.2 \mathrm{~mm}$ and $4.0 \mathrm{~mm}$. The amount of EPS added to the polyurethane reaction mixture was in the range of 6 to $150 \mathrm{wt} \%$ in relation to polyurethane reagents. The idea of this process is co-expansion of EPS and PUR and full energy balance. EPS is expanded by using the heat of exothermic polyol and isocyanate reaction. This technology allows to create a new composite material based on well-known and widely applied polymers. The major problem in obtaining PSUR composite is associated with the nature of the polymerization process, i.e., polyurethane exothermic polymerization reaction, which "drives" the endothermic process of growth of EPS beads. Both processes have different dynamics. Too small amount of heat emitted during the polymerization of PUR does not lead to the growth of EPS beads. On the other hand, too high temperature of the process will exceed the temperature of degradation of the polystyrene beads. For the purpose of the study, a concept of the heat density of PUR foaming process $q=\int_{0}^{t_{k}} \dot{q}(t) d t$ was introduced and it is defined as the amount of heat (expressed in $\mathrm{J}$ ) per unit area (in $\mathrm{m}^{2}$ ) emitted during the first $600 \mathrm{~s}$ of the PUR foaming process. Using the trial-and-error method it was demonstrated that this is enough time to observe all the processes that occur in the foam, from the moment of pouring the PUR compound into the mold, until the aging and cross-linking of the foam structure. 
For the purpose of the study two PUR systems were selected: one with high and one with low heat density of the foaming process $q$.

The aim of this work was to examine the impact of the EPS addition on the heat flux density $\dot{q}(t)$ of the foaming process of polyurethane-polystyrene porous composite (PSUR), to describe the dynamics of this process during first $600 \mathrm{~s}$, as well as to estimate volume fractions of EPS phase in the PSUR composite.

\section{Experimental}

\subsection{Materials}

There are many polyurethane systems that can be applied as thermal insulating materials in buildings or used for any industry applications. These systems differ in thermal conductivity values and densities, and, most importantly, they differ in heat densities of foaming process [14]. For our studies we decided to choose only two of them, one with high and one with low heat density of foaming process $q$. The aim of the experiment was to obtain a composite foam of lower thermal conductivity than EPS.

The materials used for the preparation of the composite are listed below:

- Owipian ${ }^{\circledR} 1325$ and Owipian ${ }^{\circledR} 0308$ (Synthos S.A., Oświęcim, Poland), both in the form of polystyrene beads with main particle size: $1.60 \mathrm{~mm}$ to $2.40 \mathrm{~mm}$ (Owipian ${ }^{\circledR} 1325$ ) and 0.4 to $0.7 \mathrm{~mm}$ (Owipian ${ }^{\circledR}$ 0308), containing 5 to $6 \mathrm{wt} \%$ of pentane isomers as an blowing agent and hexabromocyclododecane (up to 0.5 wt $\%$ ) which reduces flammability of the foam.

- IZOPIANOL 22/33 OT-P (product of Purinova Sp. z o. o., Tarnów, Poland), which was a two-component system for the production of rigid polyurethane foam used for insulation. Component A was a mixture of polyols, catalyst, silicone surfactant and HFC 365/225 (1,1,1,3,3-pentafluorobutane) as a blowing agent. Component $\mathrm{B}$ was a monomer and polymer mixture of methylene- $4,4^{\prime}$-diphenyl diisocyanate ( $30 \%$ to $32 \%$ of NCO group). Both components were mixed with a mass ratio of $\mathrm{A}: \mathrm{B}=100: 130$ (start time $18 \mathrm{~s}$ to $24 \mathrm{~s})$.

- HIT-2 (product of Prodex System Sp. z o. o., Warszawa, Poland), which was a two-component nonstandard system prepared on demand as a low-reactive polyurethane system only for tests. Component A was a mixture of polyols, catalyst, silicone surfactant and HFC 365/225 (1,1,1,3,3-pentafluorobutane) as a blowing agent. Component $\mathrm{B}$ was a monomer and polymer mixture of methylene$4,4^{\prime}$-diphenyl diisocyanate (30 to $32 \%$ of NCO group). Both components were mixed with a mass ratio $\mathrm{A}: \mathrm{B}=100: 140$ (start time $35 \mathrm{~s}$ ).

\subsection{Foam sample preparation}

The PSUR composites were synthesized using the one-shot method described in [1214]. The PUR system (IZOPIANOL or HIT-2) was mixed with the EPS beads. Then, the mixture was vigorously stirred at $1200 \mathrm{rpm}$ for $10 \mathrm{~s}$. After mixing, the mixture was poured into the $300 \mathrm{~mm} \times 300 \mathrm{~mm} \times 50 \mathrm{~mm}$ metal mold which was at room 
temperature [14]. The mold was equipped with a heating/cooling system in the form of a copper spiral on the casing of the mold. Water at a temperature controlled by refrigeration bath circulator was used as a cooling medium. After filling the mold with a PSUR mixture, the lid of the mold was closed. ALMEMO 2590-4s V5 meter equipped with a heat flux sensor (the flexible heat flow plate) type 150-1 (particularly suitable for high temperature) manufactured by AHLBORN (Germany) was used for measuring the heat flux density as a function of time. The heat flux sensor had the following dimensions: $100 \mathrm{~mm} \times 180 \mathrm{~mm} \times 0.6 \mathrm{~mm}$. The dimensions of the test field were $90 \mathrm{~mm} \times 170 \mathrm{~mm}\left(0.0153 \mathrm{~m}^{2}\right)$. The temperature of the heat flux sensor reached $160^{\circ} \mathrm{C}$. In order to not exceed this temperature, all of the heat flux density measurements were taken with the mold temperature equal to room temperature. The heat flux sensor was located on the bottom of the mold base [16]. The heat flux sensor wires were led from the mold side, and the mold side hung on hinges. The heat flux sensor was not glued to the base of the mold. Good thermal contact with the foam was assured by pouring the PSUR mixture directly on the plate heat surface. The reaction mixture was prepared in such a way that after foaming, it filled the entire mold with a volume of $0.0045 \mathrm{~m}^{3}$. To achieve that, about $180 \mathrm{~g}$ of PUR system and the fraction of EPS were added to the container. Then, the whole mixture was poured on the ALMEMO heat flux sensor. The mold was closed for the duration of the PSUR foaming process. The $\dot{q}(t)$ was recorded from the moment of pouring the reaction mixture into the mold, throughout the heating process, until cooling, i.e., between the $0 \mathrm{~s}$ and the $600 \mathrm{~s}$. Then, foams were cured in the mold for $30 \mathrm{~min}$ in order to get dimensionally stable products. After that, foams were removed from the mold and conditioned for $24 \mathrm{~h}$ at the room temperature, before being cut into appropriate specimens for testing volume fractions of EPS phase in PSUR foams as a function of EPS mass fractions.

\subsection{Methods of testing}

- The effects of EPS mass fraction (MEPS/PSUR) on the PSUR foaming process, i.e., the dependence $\dot{q}(t)$, were examined. For the IZOPIANOL 22/33 OT-P system (PUR 22/33), the heat density of the foaming process was $q_{22 / 33}=167.1 \mathrm{~kJ} / \mathrm{m}^{2}$, while for the HIT-2 system (PUR HIT-2) it was $q_{\mathrm{HIT}-2}=103.9 \mathrm{~kJ} / \mathrm{m}^{2}$. The length of each measurement $\dot{q}(t)$ was $t_{k}=600 \mathrm{~s}$. This was sufficient time to observe all the processes in the foam, from the moment of pouring the PUR mixture into the mold, i.e., for $t=0 \mathrm{~s}$, until the period of foam structure "maturation" and its cross-linking. The same measurement time was applied in all studies concerning the PSUR composite samples.

- Volume fractions of EPS phase in PSUR foams ( $\mathrm{V}_{\text {EPS/PSUR }}$ ) were calculated according to the share of the mass fractions of EPS in these foams. Program Struktura version 1 by Szulc (AGH, Poland) was used for the analysis of the volume fractions of the EPS phase in the PSUR foams. The program is based on the Cavalieri-Haquert principle. The program operates on the basis of the image binarization, so that each of the phases is represented by a different color (white: phase EPS, black: phase PUR). The calculation of phase volume fractions is performed 
Table 1 Mass fractions of EPS (FS 1325 or FS 0308) in composites PSUR, e.g.,

MEPS/PSUR and apparent density o of that foams (PUR 22/33 or PUR HIT-2)

\begin{tabular}{lll}
\hline Mass $(\mathrm{g})$ & M $_{\text {EPS } / \text { PSUR }}(\%)$ & $\varrho \pm 0.2\left(\mathrm{~kg} / \mathrm{m}^{3}\right)$ \\
\hline 22.5 & 11 & 45 \\
45.0 & 20 & 50 \\
67.5 & 27 & 55 \\
90.0 & 33 & 60 \\
112.5 & 38 & 65 \\
135.0 & 43 & 70 \\
157.0 & 47 & 75 \\
180.0 & 50 & 80 \\
202.5 & 53 & 85 \\
225.0 & 56 & 90 \\
247.5 & 58 & 95 \\
270 & 60 & 100
\end{tabular}

by counting black and white pixels in relation to the number of all pixels in the analyzed image. Samples for $\mathrm{V}_{\mathrm{EPS} / \mathrm{PSUR}}$ analysis were cut out with a bandsaw from cuboids with dimensions $300 \mathrm{~mm} \times 300 \mathrm{~mm} \times 50 \mathrm{~mm}$. The analyzed crosssectional plane with dimensions $300 \mathrm{~mm} \times 50 \mathrm{~mm}$ were taken from the middle of cuboids.

- The core apparent density of the PSUR composites was determined in accordance with the ISO 845 standard using cubical samples of $50 \mathrm{~mm}$ side length [17].

- Thermal conductivity $k$ of the PSUR composites in mass function of EPS was measured using the FOX 314 apparatus according to the PN-EN 12667 standard with the use of samples $50 \mathrm{~mm}$ thick [18].

\section{Results and discussion}

As it was mentioned above, the PSUR composites were prepared in order to estimate the influence of EPS on $\dot{q}(t)$ and on the volume fractions of EPS phase in PSUR foams $\mathrm{V}_{\mathrm{EPS} / \mathrm{PSUR}}$ as a function of mass fractions of EPS, i. e. MEPS/PSUR. The addition of EPS as a filler to PUR made it possible to obtain products that differ in density, dimensional stability (as compared to the pure PUR) and thermal conductivity [15]. The density of PSUR is considerably higher than that of PUR. The EPS was expanded by using heat from exothermic polyol and isocyanate reaction; therefore, the density of the composite filled with EPS was increasing. The thermal conductivity of PSUR composites places them between polystyrene foams (EPS), the so-called Styrofoams, and polyurethane foams (PUR) [13,15]. The mass fraction of EPS in PSUR foam, which is the same for both PUR systems (PUR 22/33 or PUR HIT-2), is shown in Table 1.

Figure 1 presents comparison of dependences $\dot{q}(t)$ for PUR 22/33 (upper figure) or PUR HIT-2 (lower figure) - both composites contained the EPS Owipian FS 1325 for $\mathrm{M}_{\mathrm{EPS} / \mathrm{PSUR}}=20 \%$. For each PUR system the difference $\Delta \dot{q}(t)=\dot{q}_{\mathrm{PUR}}(t)-$ $\dot{q}_{\text {PUR }}$ EPS $(t)$ was obtained. At the current stage of research, there are problems with 


\section{$\dot{\mathrm{q}}, W / \mathrm{m}^{2}$}

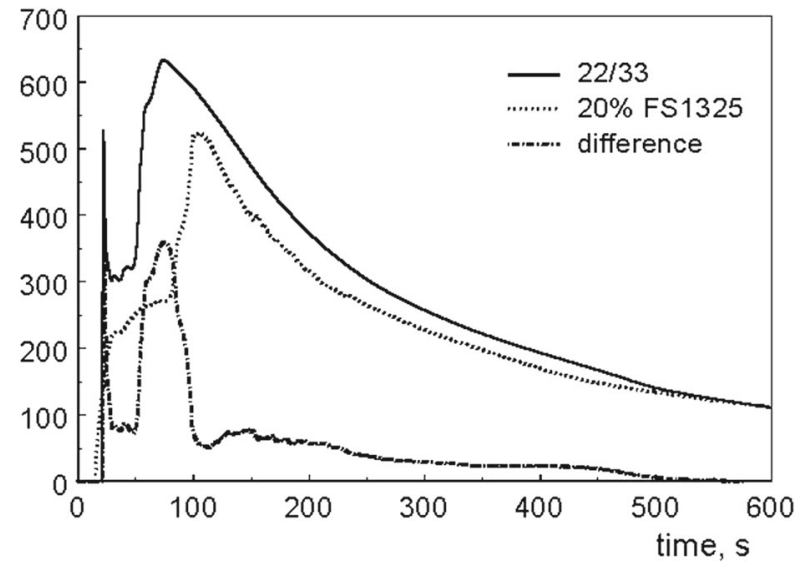

$\dot{\mathrm{q}}, W / \mathrm{m}^{2}$

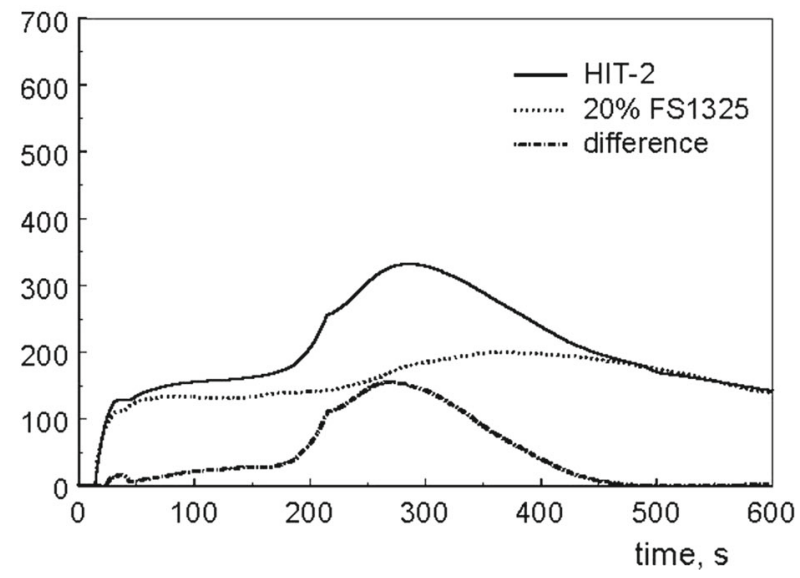

Fig. 1 Difference $\Delta \dot{q}(t)=\dot{q}_{\text {PUR }}(t)-\dot{q}_{\text {PUR }+ \text { EPS }}(t)$ for PUR 22/33 (upper figure) and PUR HIT-2 (lower figure) for $\mathrm{M}_{\mathrm{EPS} / \mathrm{PSUR}}=20 \%$

synchronization signal $\dot{q}(t)_{\text {PUR }}$ with $\dot{q}(t)_{\text {PUR }+ \text { EPS }}$. Therefore, a possible integration of the function $\Delta \dot{q}(t)$ may be subject to a small error, which is difficult to estimate.

The measurement results of $\dot{q}(t)$ obtained in the PSUR foaming process based on the PUR 22/33 and on the PUR HIT-2 - both composites contained the Owipian FS 1325 fraction-are shown in Figs. 2 and 3.

Calculation of the foaming process heat density of PSUR $q_{\text {PUR }}+$ EPS composites based on both PUR systems as a function of mass fraction of EPS FS 1325 and EPS FS0308 is shown in Fig. 4.

Foaming polystyrenes (EPS) in the form of fillers to PUR systems induce linear load power of these systems. This load is independent of the type of EPS fraction (FS1325 or FS0308) and also of the PUR system (the angle of the curves with respect to the $x$-axis is similar). 


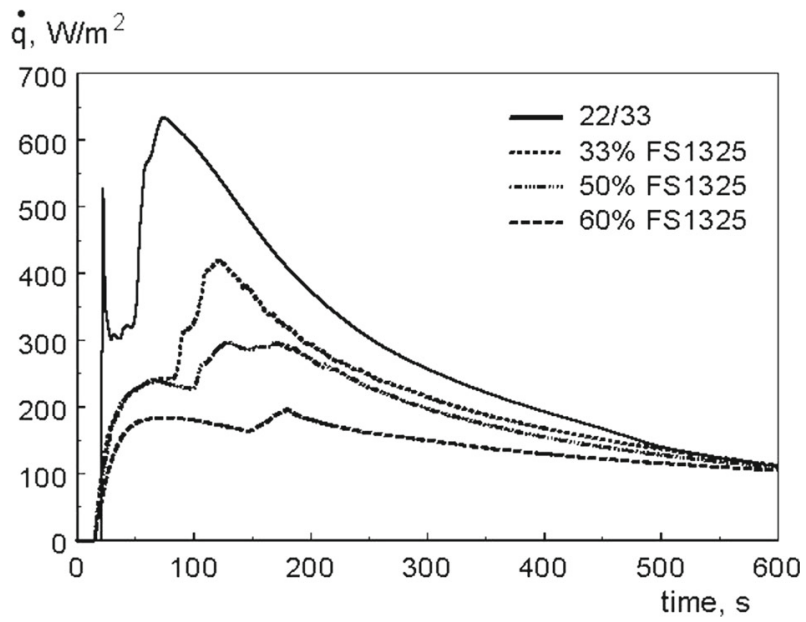

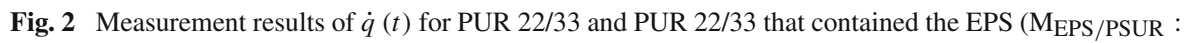
$33,50,60 \%$ )

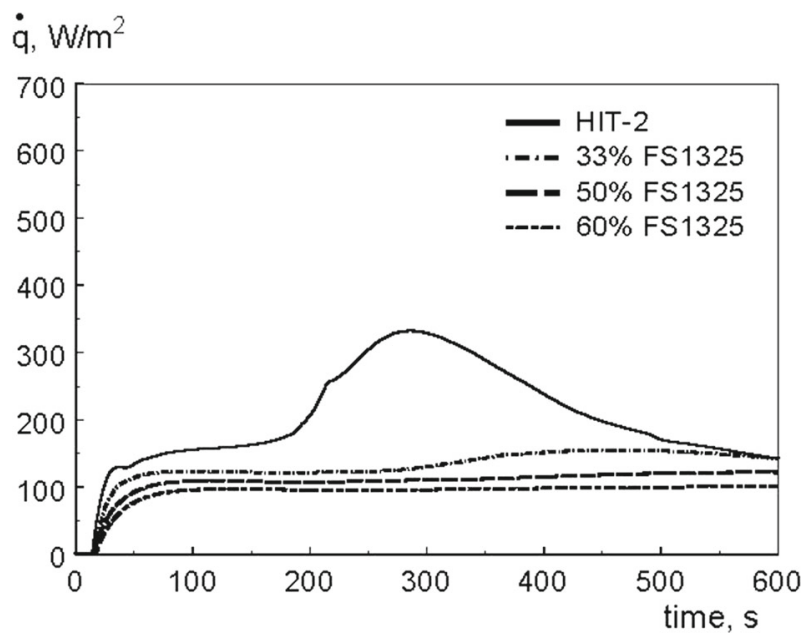

Fig. 3 Measurement results of $\dot{q}(t)$ for PUR HIT-2 and PUR HIT-2 containing the EPS (MEPS/PSUR : $33,50,60 \%$ )

Analysis of volume fractions of EPS in PSUR foams $V_{\text {EPS/PSUR }}(\%)$, is shown in Fig. 5. Due to the difficulties in distinguishing between the correct phase of EPS and noise during PSUR foams image binarization with addition of fraction FS 0308, the analysis of $V_{\text {EPS/PSUR }}$ shares is limited to fraction FS 1325.

Figure 5 shows that with small EPS mass additions (MEP/PSUR: $10 \%$ to $30 \%$ )), a bigger volume fraction of the polystyrene phase $\left(V_{\mathrm{EPS} / \text { PSUR }}\right)$ is attributable to materials obtained from PUR HIT-2 system, which results from a lack of thermal degradation of the beads in the materials. In this range thermal degradation of the EPS beads occurs in PSUR foams prepared from PUR 22/33 system. By adding EPS, we introduce 


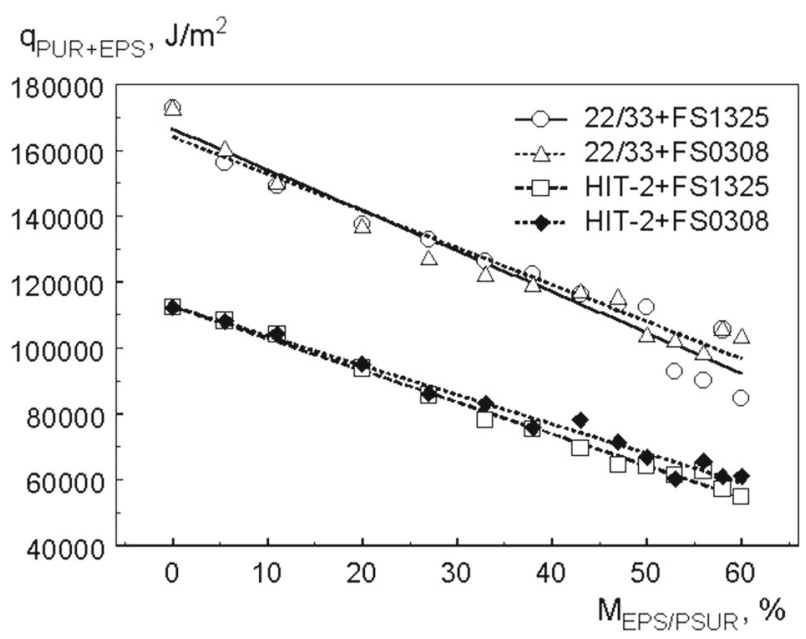

Fig. 4 Summary of the heat density of the PSUR foaming process as a function of the mass fraction of EPS

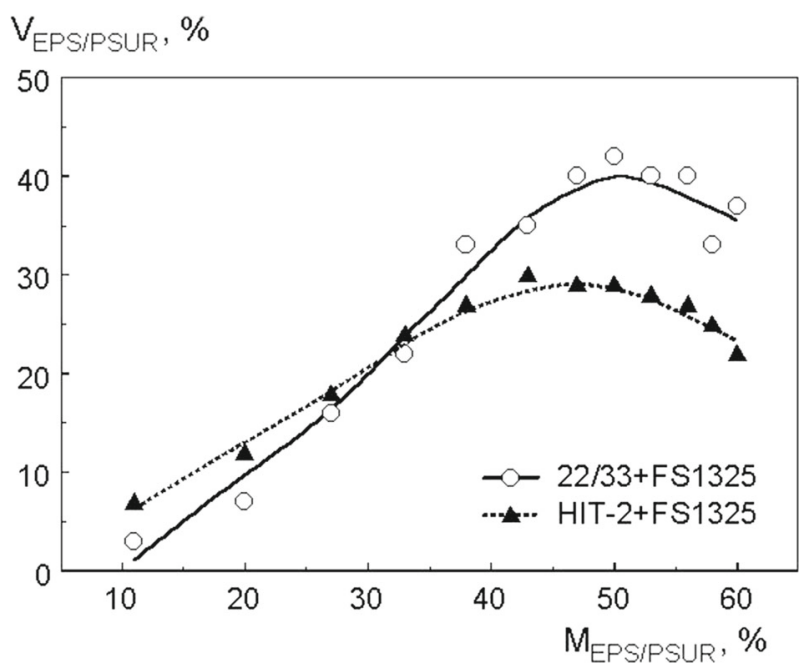

Fig. 5 Summary of the volume fraction $V_{\text {EPS/PSUR }}$ of PSUR foams as a function of the mass fraction MEPS/PSUR of EPS FS 1325

the heat-absorbing medium. The more the EPS beads, the more we reduce the heat density of the PUR foaming process. In the case of PUR HIT-2 system, the generated energy is much smaller than in the case of PUR 22/33 system. Above the threshold of $\mathrm{M}_{\mathrm{EPS} / \mathrm{PSUR}}=30 \%$ for the EPS addition, the PSUR foam, based on PUR HIT-2, contains $27 \%$ of EPS volume fraction, i.e., $6 \%$ less, than in the case of high-energy system based on PUR 22/33. As a result, after crossing the barrier of polystyrene phase degradation, which for a PSUR foam, based on PUR 22/33, is in the range of $\mathrm{M}_{\mathrm{EPS} / \mathrm{PSUR}}$ : 10 to $30 \%$ of EPS mass fraction addition, the high-energy system 


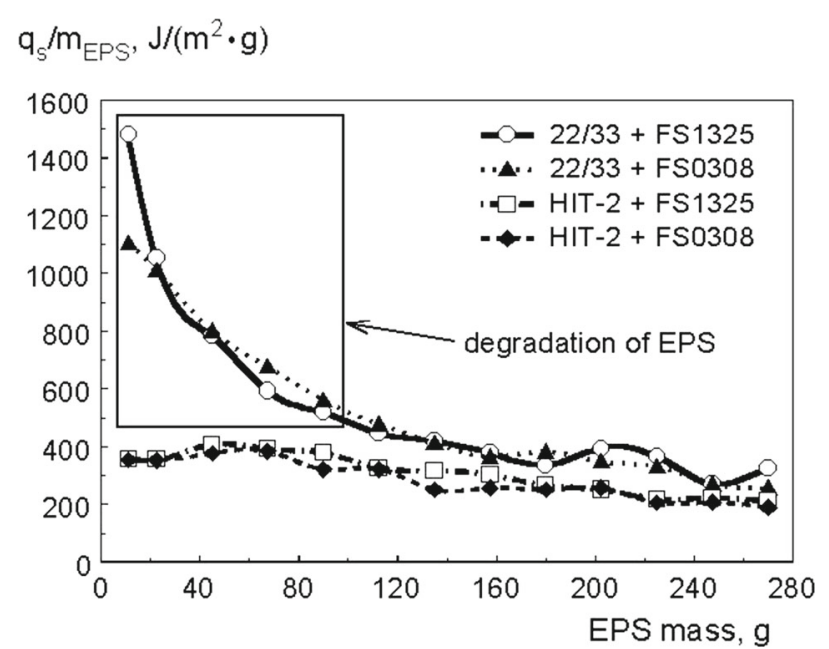

Fig. 6 Selection of the heat density loss of PUR 22/33 and PUR HIT-2 systems calculated for $1 \mathrm{~g}$ of EPS in mass function of EPS: FS 1325 and FS 0308

generates more energy for growth of beads than the low-energy system. As a result, the obtained EPS beads are bigger. For the EPS mass fraction of above $50 \%$ for the PUR 22/33 and PUR HIT-2 system, the amount of energy for beads' growth is too small and despite the increase in the EPS mass fraction in PSUR foam, the EPS volume fraction in PSUR foam, i.e., $V_{\text {EPS/PSUR }}$, decreases. Additionally, a much lower degree of expansion of EPS fraction is obtained in PSUR foam.

The heat density loss of the PSUR foaming process as a result of adding EPS fraction, calculated for $1 \mathrm{~g}$ of the EPS additive, is:

$$
\frac{q_{s}}{m_{\mathrm{EPS}}}=\frac{q_{\mathrm{PUR}}-q_{\mathrm{PUR}+\mathrm{EPS}}}{m_{\mathrm{EPS}}}, \frac{\mathrm{J}}{\mathrm{m}^{2} \cdot \mathrm{g}} .
$$

Dependencies $\frac{q_{s}}{m_{\mathrm{EPS}}}$ in a function of EPS mass for both PUR systems are shown in Fig. 6.

The PUR 22/33 system, as opposed to the PUR HIT-2 system, has a high heat density value $q$. Thus, during the foaming process in the PSUR foam, which is obtained on the basis of PUR 22/33 system, temperatures are above $95^{\circ} \mathrm{C}$. Therefore, in the range of EPS mass of between $22.5 \mathrm{~g}$ and $112.5 \mathrm{~g}$, a degradation of EPS fraction is observed. The PUR HIT-2 system, due to the small value of $q$ over the range of EPS mass, does not degrade beads. Thus, it is possible to obtain PSUR foams with small EPS mass fractions.

Figure 7 presents a comparison of dependencies $k$ (EPS mass) of the PSUR foams (PUR 22/33) in mass function of EPS (fraction: FS 1325 or FS 0308). Curves $k$ (EPS mass) can be divided into two areas:

(a) $22.5 \mathrm{~g}$ to about $120 \mathrm{~g}$ - an increase in thermal conductivity $k$ is associated with the degradation of EPS beads. The beads are replaced by holes filled with pairs 


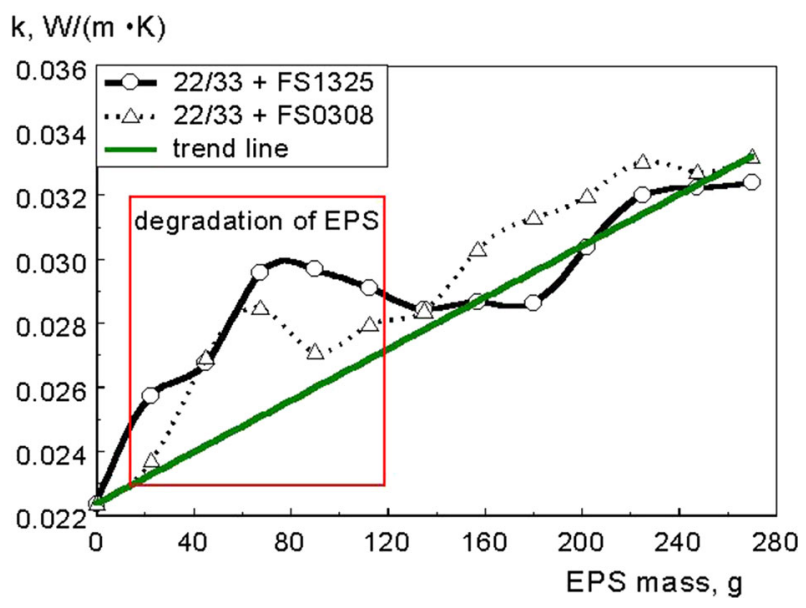

Fig. 7 Summary of the thermal conductivity of the PSUR foams (PUR 22/33) in mass function of EPS (FS 1325 and FS 0308)

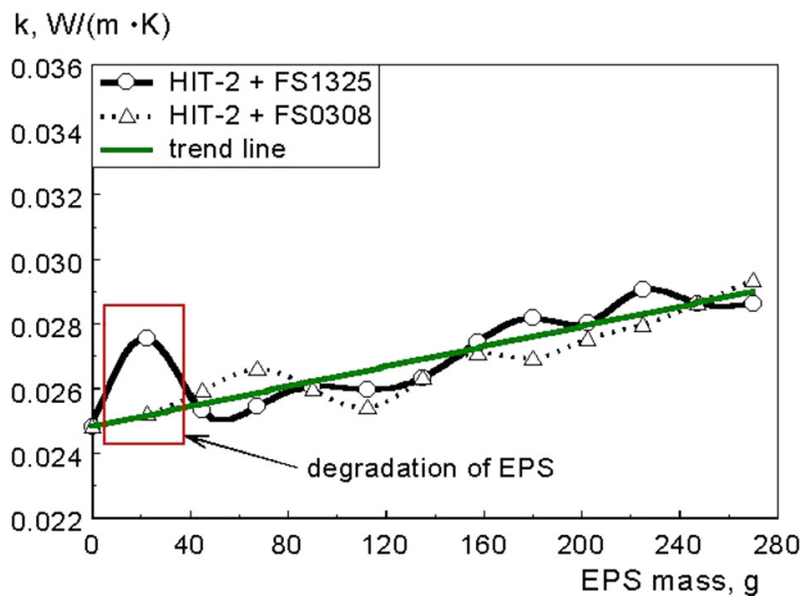

Fig. 8 Summary of the thermal conductivity of the PSUR foams (PUR HIT-2) in mass function of EPS (FS 1325 and FS 0308)

of blowing agents. Convective heat transfer in the holes increases the thermal conductivity $k$ of the PSUR composite;

(b) about $120 \mathrm{~g}$ to $270 \mathrm{~g}$-an increase in thermal conductivity $k$ is caused by an increase in the volume of the EPS beads in the composite. The more the developed EPS beads in the composite structure of PSUR, the closer its thermal conductivity to the thermal conductivity of an expanded polystyrene $\left(k=0.037 \mathrm{~W} \cdot \mathrm{m}^{-1} \mathrm{~K}^{-1}\right)$. The differences between the curves $k$ (EPS mass) formed by adding either FS 1325 or FS 0308 EPS result from the fact that the fraction with smaller EPS beads, i.e., FS 0308, formed in result of degradation gives rise to much smaller holes than fraction FS 1325 . For this reason, an increase in thermal conductivity $k$ by convection in such composite was smaller than in composite with fraction FS 1325; 
Figure 8 presents a comparison of dependencies $k$ (EPS mass) of the PSUR foams (HIT-2) in mass function of EPS (fraction: FS 1325 and FS 0308). The growth trend of thermal conductivity $k$ in the mass function of the EPS (FS 1325 and FS 0308) is similar as in PSUR foams based on PUR 22/33 system. However, for low-energy system, like the PUR HIT-2, degradation of EPS beads occurs only with the addition of EPS fraction equal to $22.5 \mathrm{~g}$. In the remaining range of EPS additive, i.e., to $270 \mathrm{~g}$, a smaller share of volume fraction of EPS in the structure of the PSUR composite makes the thermal conductivity closer to the thermal conductivity of pure foam HIT-2 $\left(k=0.025 \mathrm{~W} \cdot \mathrm{m}^{-1} \mathrm{~K}^{-1}\right)$.

\section{Conclusion}

The obtained results prove that it is possible to modify the PUR, foamed by the chemical blowing agent with the addition of EPS beads. This process can be observed with the use of the ALMEMO meter, which is equipped with a heat flux sensor 150-1 for measuring the heat flux density as a function of time. It seems that among the many devices of this type, the optimum heat meter was chosen, i.e., the one that uses the full range of $\dot{q}(t)$ and full range of temperature, i.e., up to $160^{\circ} \mathrm{C}$. The location of the heat flow meter sensor at the bottom of the mold for PSUR foaming was extremely useful for achieving the objectives of the work [16]. Measurements of $\dot{q}(t)$ revealed the dynamics of the polymerization of PUR and PSUR foams as well as allowed to sort the various PUR systems in the amount of heat released in the process of polymerization of these foams [14]. The PSUR composites are cheaper than polyurethane foams and can have industrial application.

Thermal conductivity measurements of the PSUR foams, based on the PUR 22/33 or PUR HIT-2 system, in mass function of EPS (FS 1325 or FS 0308) have shown that we can model the thermal insulation properties of such composites in two ways: by the selection of PUR system, taking into consideration the amount of heat generated in relation to the weight fraction of EPS, and by the selection of EPS fraction, taking into consideration the size of EPS beads.

On the basis of Fig. 5 it can be concluded that the PUR 22/33 system generates beads of $10 \%$ bigger volume fraction of the polystyrene phase ( $V_{\text {EPS }} /$ PSUR $)$ within the range of $40-50 \%$ of $M_{E P S}$ PSUR. It means that during the free growth of such a system the usage of more expensive polyurethane system will be lower, and therefore, the cost of PSUR composite will also be lower. In Figs. 7 and 8 the thermal conductivity of the PSUR foams (PUR 22/33 and HIT-2) are presented in mass function of EPS (FS 1325 and FS 0308). The thermal conductivity of the PSUR based on the PUR $22 / 33$ is greater than the one of HIT-2, but their values are still lower than the thermal conductivity of EPS.

Open Access This article is distributed under the terms of the Creative Commons Attribution 4.0 International License (http://creativecommons.org/licenses/by/4.0/), which permits unrestricted use, distribution, and reproduction in any medium, provided you give appropriate credit to the original author(s) and the source, provide a link to the Creative Commons license, and indicate if changes were made. 


\section{References}

1. T. Whelan, Polymers Technology Dictionary (Chapman \& Hall, London, 1994), p. 493

2. G. Wypych, Handbook of fillers (ChemTecPublishing 2010), pp.13-266

3. W.H. Lee, S.W. Lee, T.J. Kang, K. Chung, Jae Ryoun Youn, Fibers Polym. 3, 159 (2002)

4. L. Song, Y. Hu, B. Li, S. Wang, W. Fat, Int. J. Polym. Anal. Charact. 8, 317 (2003)

5. B. Czupryński, J. Liszowska, J. Paciorek-Sadowska, Polimery 53, 133 (2008)

6. H. Hatakeyema, N. Tanamachi, H. Matsumura, S. Hirose, T. Hatakeyam, Thermochim. Acta 431, 155 (2005)

7. F. Saint-Michel, L. Chazeau, J. Cavaille, Compos. Sci. Technol. 66, 2709 (2006)

8. M. Pinto, S. Silva, L. Carvalho, J. Almeida, Int. J. Polym. Mater. 56, 885 (2007)

9. W. Zatorski, Z. Brzozowski, A. Kolbrecki, Polym. Degrad. Stab. 93, 2071 (2008)

10. Polish Pat. 328594 (2000)

11. P. Pat, Applied 395, 886 (2011)

12. A. Sabanowska, E. Malewska, J. Polaczek, Mod. Polym. Mater. Environ. Appl. 4, 107 (2010)

13. M. Trzyna, P. Koniorczyk, J. Zmywaczyk, Ciepłownictwo Ogrzewnictwo Wentylacja 4, 141 (2011)

14. M. Trzyna, B. Zygmunt, P. Koniorczyk, J. Zmywaczyk, M. Preiskorn, Przem. Chem. 92, 975 (2013)

15. E. Malewska, M. Trzyna, A. Sabanowska, A. Prociak, P. Koniorczyk, Polimery 56, 865 (2011)

16. T.E. Diller, Heat flux measurement, Ch. 18, in Handbook of Measurement in Science and Engineering, ed. by M. Kutz (Wiley, Hoboken, 2013), pp. 629-659

17. PN-EN ISO 845 (2010)

18. PN-EN 12667 (2002)

19. A. Biedermann, C. Kudoke, A. Merten, E. Minogue, U. Rotermund, H.P. Ebert, U. Heinemann, J. Fricke, H. Seifert, J. Cell. Plast. 37, 467-483 (2001)

20. W.-H. Tao, H.-C. Hsu, C.-C. Chang, C.-L. Hsu, Y.-S. Lin, J. Cell. Plast. 37, 310-332 (2001) 